\title{
Striatal Functional Alteration in Adolescents Characterized by Early Childhood Behavioral Inhibition
}

\author{
Amanda E. Guyer, ${ }^{1}$ Eric E. Nelson, ${ }^{1}$ Koraly Perez-Edgar, ${ }^{1,7}$ Michael G. Hardin,,${ }^{1,2}$ Roxann Roberson-Nay, ${ }^{3}$ \\ Christopher S. Monk, ${ }^{4}$ James M. Bjork, ${ }^{5}$ Heather A. Henderson, ${ }^{6}$ Daniel S. Pine, ${ }^{1}$ Nathan A. Fox, ${ }^{2}$ and Monique Ernst ${ }^{1}$ \\ ${ }^{1}$ Emotional Development and Affective Neuroscience Branch, National Institute of Mental Health, National Institutes of Health (NIH), Bethesda, Maryland \\ 20892, ${ }^{2}$ Department of Human Development, University of Maryland, College Park, Maryland 20742, ${ }^{3}$ Department of Psychiatry, Virginia Commonwealth \\ University, Richmond, Virginia 23284, ${ }^{4}$ Department of Psychology, University of Michigan, Ann Arbor, Michigan 48109, ${ }^{5}$ Laboratory of Clinical and \\ Translational Studies, National Institute on Alcohol Abuse and Alcoholism, NIH, Bethesda, Maryland 20892, ${ }^{6}$ Department of Psychology, University of \\ Miami, Coral Gables, Florida 33146, and ${ }^{7}$ Department of Psychology, George Mason University, Fairfax, Virginia 22030
}

The temperamental style of behavioral inhibition has been characterized by exaggerated behavioral and neural responses to cues signaling threat. Virtually no work, however, has addressed whether behavioral inhibition may also confer heightened brain activation in response to positively valenced incentives. We used event-related functional MRI (fMRI) and a monetary incentive delay task to examine whether the neural response to incentives is also greater in adolescents characterized as behaviorally inhibited early in life compared with those characterized as non-inhibited. Whereas task performance did not differ between groups, fMRI revealed greater striatal activation to incentives in behaviorally inhibited adolescents than in non-inhibited adolescents. This was regardless of whether the incentive was an anticipated gain or loss. Alteration in neural systems underlying behavior modulated by both negative and positive contingencies may represent a correlate of behavioral inhibition that also underlies vulnerability to various forms of developmental psychopathology.

Key words: fMRI; striatum; reward; adolescence; motivation; temperament

\section{Introduction}

Temperament refers to stable individual differences in psychological and physiological responsivity to stimuli (Rothbart et al., 2001). Perhaps the best understood temperamental construct is behavioral inhibition (Fox et al., 2005). Behaviorally inhibited children exhibit heightened vigilance, negative affect, and reactivity to novelty compared with non-inhibited counterparts (Kagan et al., 1988a; Kagan and Snidman, 1991). Physiological patterns further differentiate inhibited from non-inhibited children, e.g., elevated baseline cortisol, higher heart rate, and right frontal electroencephalogram asymmetry (Kagan et al., 1987; Calkins et al., 1996; Fox et al., 1996).

Although attentional aspects of behavioral inhibition traditionally have been attributed to enhanced amygdala reactivity in response to cues that provoke withdrawal (Kagan et al., 1987; Schwartz et al., 2003), virtually no research on behavioral inhibition has examined attention to cues that engage approach behaviors. Such work is important because behavioral inhibition could be associated with biased responses to both rewards and punishments (Reynolds and Berridge, 2002; Roitman et al., 2005). In a

\footnotetext{
Received Feb. 15, 2006; revised April 24, 2006; accepted May 8, 2006.

This work was supported in part by the Intramural Research Program of the National Institutes of HealthNational Institute of Mental Health and by National Institute of Child Health and Development Grant HD 17899 (N.A.F.). We thank Harvey Iwamoto and Stacey Barton for technical support.

Correspondence should be addressed to Dr. Amanda E. Guyer, 15K North Drive, Room 208, Bethesda, MD 208922670. E-mail: amandaguyer@mail.nih.gov.

DOI:10.1523/JNEUROSCI.0666-06.2006

Copyright $\odot 2006$ Society for Neuroscience $\quad$ 0270-6474/06/266399-07\$15.00/0
}

preliminary study, we compared performance on the monetary incentive delay (MID) task (Knutson et al., 2001a) in college students categorized by shyness (Hardin et al., 2006). Results showed faster responses to incentives in shy versus not shy students, interpreted as enhanced reward sensitivity. Several functional magnetic resonance imaging (fMRI) studies using the MID task implicate striatal circuitry in psychological processes engaged by monetary incentives (Knutson et al., 2000, 2001a,b; Bjork et al., 2004). Specifically, such work demonstrates striatal activation to cues that elicit anticipation of monetary incentives and enhanced motor performance (Knutson et al., 2000). Thus, our behavioral findings suggest that neural correlates of behavioral inhibition may be more widespread than previously described.

The present study uses fMRI and the MID task in a unique cohort of adolescents characterized since infancy on temperament measures. Neural activation was examined in striatal components based on regional specialization in incentive processing. For example, nucleus accumbens and caudate are implicated in coding cue properties, and putamen is involved in motor response (Berns et al., 2001; Knutson et al., 2001a; Delgado et al., 2003; O'Doherty et al., 2004; Zink et al., 2004; Daw et al., 2005; Haruno and Kawato, 2006). Regionally parsing the striatum is important because the MID involves processing cue salience and relating salience to action. We also examined amygdala activation based on evidence supporting the role of the amygdala in response to rewards as well as to negative stimuli (Baxter et al., 2000; Breiter et al., 2001; Schwartz et al., 2003; Ernst et al., 2005) 
and as a theorized mediator of inhibited behavior (LeDoux et al., 1988; Kagan, 1994).

We hypothesized that behaviorally inhibited adolescents would demonstrate greater striatal activation than non-inhibited adolescents to increasing monetary incentives. This hypothesis was based on our past behavioral work in college students (Hardin et al., 2006) and children (Henderson, 2003). Given the putative role of the amygdala in anxiety and behavioral inhibition (Schwartz et al., 2003), we also hypothesized that inhibited versus non-inhibited adolescents would show enhanced amygdala activation for potential monetary loss.

\section{Materials and Methods}

Participants. Adolescents were drawn from cohorts participating in a longitudinal study of temperament and affect regulation. At 4 months of age, 433 subjects were screened for motoric and emotional reactivity to novel visual and auditory stimuli (Kagan and Snidman, 1991; Calkins et al., 1996). Of the 433 infants screened, 153 with reactivity scores at the high and low extremes were selected for inclusion in a longitudinal study of temperament. At ages 9 months, 14 months, 24 months, 4 years, and 7 years, electroencephalogram data, parent-reported temperament surveys, and observations of children's reactions to unfamiliar stimuli and peers were collected (Calkins et al., 1996; Fox et al., 2001). Previous analyses involving these children up to 4 years of age has been described by Fox et al. (2001) and Henderson et al. (2004).

Of the 153 children followed longitudinally, 44 were recruited in adolescence (ages 10-15 years) to participate in the present study. The 44 children were selected based on their degree of behavioral inhibition as determined by a composite of assessments collected in childhood. The behavioral inhibition composite included laboratory measures of social reticence, maternal ratings of shyness from the Colorado Child Temperament Inventory (Buss and Plomin, 1984), and maternal ratings of internalizing problems from the Child Behavior Checklist (Achenbach et al., 1991). To ensure that the children selected for the current study reflected the full distribution of behavioral inhibition in the larger cohort, selection began by moving inward from the two extremes and outward from the median of the composite. Scale scores of these measures were standardized and averaged to create the behavioral inhibition composite (range, -0.70 to 1.63 ; mean $\pm \mathrm{SD}, 0.07 \pm 0.65$ ). To dichotomize the behavioral inhibition composite, standardized scores of behavioral inhibition at 14 and 24 months and social reticence at 4 and 7 years were used in a cluster analysis (supplemental Table 1, available at www. jneurosci.org as supplemental material). A two-cluster solution was derived with 17 children in cluster one and 27 children in cluster two. Mean levels of behavioral inhibition and social reticence in both toddlerhood and early childhood, respectively, were higher in cluster one and were thus labeled "behaviorally inhibited" (BI); cluster two was labeled "behaviorally non-inhibited" (BN). For complete details of inhibition classification, see supplemental data 1 (available at www.jneurosci.org as supplemental material).

Of the 44 adolescents selected for the neuroimaging study, five declined participation and four met study exclusionary criteria (e.g., dental braces, severe psychopathology, use of psychoactive substance); for three, technical problems prohibited data acquisition. Adolescents who did and did not complete the scan were comparable in age $\left(t_{(42)}=0.33\right.$, NS), intelligence quotient (IQ) $\left(t_{(40)}=1.61, \mathrm{NS}\right)$, sex $\left(\chi_{(1,44)}^{2}=1.83\right.$, NS), and inhibition classification $\left(\chi_{(1,44)}^{2}=0.20\right.$, NS). Of the 32 adolescents who completed the scan, 13 were classified as BI (age, mean $\pm \mathrm{SD}$, $13.40 \pm 1.68$ years; IQ, mean $\pm \mathrm{SD}, 118.7 \pm 10.17$; sex, five male, eight female), and 19 were classified as $\mathrm{BN}$ (age, mean \pm SD, $13.26 \pm 1.80$ years; IQ, mean $\pm \mathrm{SD}, 117.74 \pm 8.41$; sex, 9 male, 10 female). The two inhibition groups did not differ in age $\left(t_{(30)}=0.22\right.$, NS), IQ $\left(t_{(29)}=0.22\right.$, NS), or sex $\left(\chi_{(1,32)}^{2}=0.25\right.$, NS).

Diagnoses of current psychiatric illness were ascertained using the Schedule for Affective Disorders and Schizophrenia for School Aged Children-Present and Lifetime Version, administered by an experienced clinician who exhibited satisfactory reliability on the exam $(\kappa>0.75$ for all diagnoses) (Kaufman et al., 1997). Four BI (31\%) and three BN (16\%) adolescents met criteria for an Axis I diagnosis. BI adolescents had either an internalizing or externalizing disorder, whereas BN adolescents only had externalizing disorders. Analyses including and those not including data from these seven adolescents generated identical conclusions. As a result, we retained these subjects in the analyses reported here.

The institutional review boards at the National Institute of Mental Health (Bethesda, MD) and the University of Maryland (College Park, $\mathrm{MD}$ ) approved this study. All subjects and their parents provided written informed assent/consent to participate in the study.

MID task. The MID task has been shown in a series of fMRI studies to consistently engage the striatum during anticipation of potential monetary gain and loss (Knutson et al., 2000, 2001a,b; Bjork et al., 2004). A parametric version of the task manipulates motivation for obtaining a gain or for avoiding a loss by varying the amount of money at stake (Knutson et al., 2001a). The MID task required participants to respond with a button press as quickly as possible during the presentation of a target. If the participants succeeded in pressing the button during target presentation, they either won or avoided losing money.

The MID task used was the same version used previously in work with both adolescents and adults. The task consisted of two runs of 72 contiguous $6 \mathrm{~s}$ trials. Each trial began with the presentation of a cue, followed by a crosshair fixation point, and finally a response target. Cues appeared for $250 \mathrm{~ms}$, whereas the fixation delay $(2000-2500 \mathrm{~ms})$ and target (160-250 $\mathrm{ms})$ each appeared at variable intervals. Circle cues $(n=64)$ indicated a potential monetary gain (reward) if the button press occurred quickly enough at target onset; square cues $(n=64)$ signified a potential monetary loss (punishment) if the button press did not occur quickly enough at target onset; and triangle cues $(n=16)$ indicated no money at stake. Magnitude of incentive values was represented by a single line $(\$ 0.20 ; n=$ $32)$, two lines ( $\$ 1 ; n=32)$, or three lines $(\$ 5 ; n=32)$ within the cue. After the disappearance of the target, feedback $(1650 \mathrm{~ms})$ notified participants of a gain, loss, or no change and indicated current, cumulative dollar amount. The order of trial type was completely random within each run. Participants were told that they would receive the dollar amount won.

Participants completed a practice session of the MID task at the start of the scan, which served two main purposes. First, it minimized learning effects on performance during scanning. Second, it provided an estimate of each subject's reaction time (RT) for standardizing task difficulty, minimizing a potential confound of large performance differences overshadowing between-group differences in brain activation. Task difficulty was tailored to each participant's ability by adjusting target duration on the actual task such that participants succeeded on $\sim 66 \%$ of their responses using mean RT and accuracy rate from the practice task (Knutson et al., 2001a).

fMRI acquisition. Scanning occurred in a General Electric (Waukesha, WI) Signa 3 tesla magnet. A Cedrus (San Pedro, CA) Lumina response box recorded behavioral data. MID stimuli were projected onto a screen at the foot of the scanner bed and viewed with mirrors mounted on the head coil. Head movement was constrained by the use of foam padding. Functional scans were acquired with the following sequence parameters. Each brain volume consisted of 30 interleaved slices $4 \mathrm{~mm}$ thick acquired in the sagittal plane using a $\mathrm{T} 2^{\star}$-weighted echo-planar sequence with a repetition time (TR) of $2500 \mathrm{~ms}$, echo time (TE) of $23 \mathrm{~ms}$, and flip angle of $90^{\circ}$. Voxel dimension was $3.75 \times 3.75 \times 4.0 \mathrm{~mm}$. Matrix size was $64 \times$ $64 \mathrm{~mm}$, and field of view (FOV) was $24 \mathrm{~cm}$. To allow for signal stabilization, four acquisitions were obtained before task onset. A high-resolution structural image was also acquired for each subject using a T1-weighted standardized magnetization prepared spoiled gradient recalled echo sequence: $1241 \mathrm{~mm}$ slices, TR of $8100 \mathrm{~ms}$, TE of $32 \mathrm{~ms}$, flip angle of $15^{\circ}$, matrix size of $256 \times 256 \mathrm{~mm}$, and FOV of $24 \mathrm{~cm}$.

fMRI data analysis. Analysis of Functional and Neural Images (AFNI) software was used to analyze fMRI data (Cox, 1996). Standard preprocessing of echo-planar data included slice time correction, motion correction, and spatial smoothing with a $6 \mathrm{~mm}$ full-width half-maximum smoothing kernel. A despiking algorithm was then applied to the data on a voxelwise basis to smooth out deviations in signal $>2.5 \mathrm{SD}$ from the mean, followed by a bandpass filtering algorithm to smooth cyclical fluctuations in signal that were not temporally indicative of a hemodynamic response (either $>0.011$ or $<0.15 \mathrm{~s}$ ). Each subject's data were converted 
to percentage signal change using each subject's voxelwise time series mean as a baseline.

Preprocessed time series data for each individual were analyzed by multiple regression (Neter et al., 1996). The regression model consisted of regressors of interest, six regressors modeling effects attributable to residual motion (using the motion correction factors in the $x, y$, and $z$ planes and in the yaw, pitch, and roll dimensions), and two regressors modeling baseline and linear trends for each of the two runs. Regressors of interest included cues signaling trial type (e.g., large, medium, and small potential gain and large, medium, and small potential loss) and were convolved with a gamma variate function that modeled a prototypical hemodynamic response (Cohen, 1997). Idealized signal time courses were estimated based on onset time of different event types during the task.

Contrasts of whole-brain blood oxygen level-dependent (BOLD) activation were created for each subject for cues signaling potential gains and potential losses of (1) large \$ versus no \$, (2) medium \$ versus no \$, and (3) small \$ versus no \$. Based on our a priori hypothesis, we compared groups on change in striatal and amygdala activity during anticipated gains and losses at different incentive magnitudes. Mean contrast values were generated for all voxels within the striatum [including nucleus accumbens, caudate (head and body), and putamen] and the amygdala. To examine the degree to which between-group differences occurred more generally throughout the brain, this method was repeated for the primary visual cortex [Brodmann area (BA) 17] and the primary motor cortex (BA4). BA17 is a region that may be affected by stimulus salience, but we expected no between-group differences in BA17 activation. BA4 is implicated in planning and executing movements, processes that engage a motor circuit encompassing the striatum. We would expect stimulus salience and group status to both potentially influence motivated behavior and thus engage BA4. Talairach anatomical boundaries provided by AFNI were used to define voxels that fell within each region of interest (ROI) after spatial normalization (Talairach and Tournoux, 1988).

The contrast values generated within each ROI from each subject were then entered into second group-level analyses. Group-level analyses were performed in SPSS (SPSS, Chicago, IL) using repeated-measures ANOVA. The model for striatal activation included group (BI, BN) as a between-subjects factor and incentive magnitude [small (\$0.20), medium (\$1), large (\$5)], valence (gain, loss), and striatal region (nucleus accumbens, caudate, and putamen) as within-subjects factors. The models for the amygdala, BA17, and BA4 included group, incentive magnitude, and valence as factors. Activation for each incentive magnitude was calculated as the net signal difference between anticipation of a small, medium, or large $\$$ amount and $\$ 0$, at the acquisition of the event-related hemodynamic response function peak. Interactions between group and characteristics of the MID task were the effects of primary interest. Because all striatal regions have been implicated differently in reward processes, we tested for an interaction between group and striatal region. If a significant group $\times$ striatal region effect was detected, we would then interpret results for each striatal component separately given regional functional specialization (Berns et al., 2001; Knutson et al., 2001a; Delgado et al., 2003; O'Doherty et al., 2004; Zink et al., 2004; Daw et al., 2005; Haruno and Kawato, 2006).

Behavioral data analysis. Level of task difficulty (ranging from 1 to 5, corresponding to five sets of target duration) was adjusted at the beginning of the task to normalize performance to $\sim 66 \%$ accuracy across participants. Mean task difficulty level was compared between groups using a $t$ test. Dependent variables included accuracy (i.e., proportion of successful button presses during target presentation), RT for successful hits (i.e., length of time between target onset and button presses leading to a hit), and postscan ratings. Accuracy and RT were analyzed separately using repeated-measures ANOVA, with group as a between-subjects factor and valence and incentive magnitude as within-subjects factors. To parallel the imaging analyses, which used $\$ 0$ trials as a baseline, response to $\$ 0$ cues was included in the models as a covariate. At the end of the task, subjects retrospectively rated how much they liked or disliked each of the six cues (large, medium, and small potential gain and large, medium, and small potential loss) on a scale from -5 (dislike very much) to +5 (like

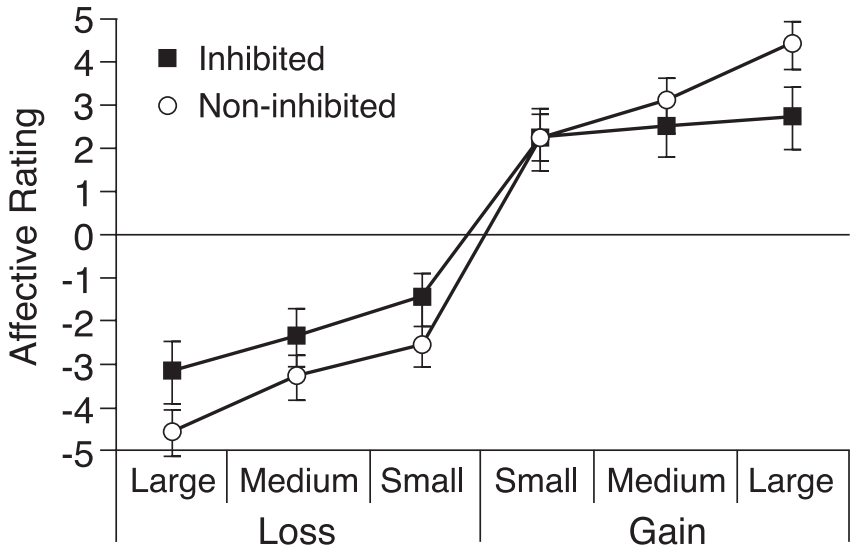

Figure 1. Between-group comparisons of postscan affective ratings of anticipation cues depicting small, medium, and large incentives for loss and gain trials. Ratings ranged from -5 (dislike very much) to +5 (like very much). Error bars represent SEM.

very much) indexing cue-elicited affective response. Affective ratings were analyzed with a similarly constructed ANOVA.

\section{Results}

\section{Behavioral data}

No significant group differences were found on any of the task performance variables. In addition, performance did not vary as a function of valence or incentive magnitude (supplemental data 2, available at www.jneurosci.org as supplemental material). Incentive values of each cue, however, influenced participants' postscan subjective ratings of cues. An interaction of valence and incentive magnitude indicated that participants' preference of cues increased as gain cue magnitude increased and decreased as loss cue magnitude increased $\left(F_{(2,50)}=12.54 ; p<0.001\right)$ (Fig. 1). This suggests that subjects had properly understood intended cue salience. Specifically, paired comparisons indicated that participants preferred $+\$ 5.00$ cues over $+\$ 1.00$ and $+\$ 0.20$ cues, and $+\$ 1.00$ cues over $+\$ 0.20$ cues (all $p$ values $<0.03$ ). In contrast, participants disliked $-\$ 5.00$ cues more than $-\$ 0.20$ cues $(p=$ 0.001 ). In addition, the absolute values of affective ratings, which indicate the intensity of emotional response to the cues, did not differ between gain cues and loss cues or between groups. Finally, a nonsignificant valence $\times$ group interaction suggests the groups did not differ by valence (gains vs losses) on the intensity of their affective response to the cues.

\section{fMRI data}

With regard to the striatum, results from the repeated-measures ANOVA indicated significant main effects of incentive magnitude $\left(F_{(2,60)}=20.20 ; p<0.001\right)$ and group $\left(F_{(1,30)}=5.16 ; p=\right.$ $0.03)$ on mean percentage signal change when collapsed across all striatal regions and both gain and loss trials. Importantly, this analysis also revealed a significant group $\times$ incentive magnitude interaction $\left(F_{(2,60)}=3.30 ; p=0.04\right)$, whereby striatal activation changed differently for the $\mathrm{BI}$ group relative to the $\mathrm{BN}$ group as incentive magnitude increased (Fig. 2). Specifically, striatal activation became stronger with larger incentive magnitudes and was larger in the BI group than in the BN group. Significant main effects of valence $\left(F_{(1,30)}=6.09 ; p=0.02\right)$ and region $\left(F_{(2,60)}=\right.$ $3.30 ; p=0.04)$ were also found for mean percentage signal change in the striatum. Striatal activation was greater during gain trials (mean $\pm \mathrm{SE}, 0.13 \pm 0.02$ ) relative to loss trials (mean $\pm \mathrm{SE}$, $0.10 \pm 0.02)$. With regard to region, significantly greater activation was found in the caudate (mean \pm SE, $0.12 \pm 0.02 ; p<.001$ ) 


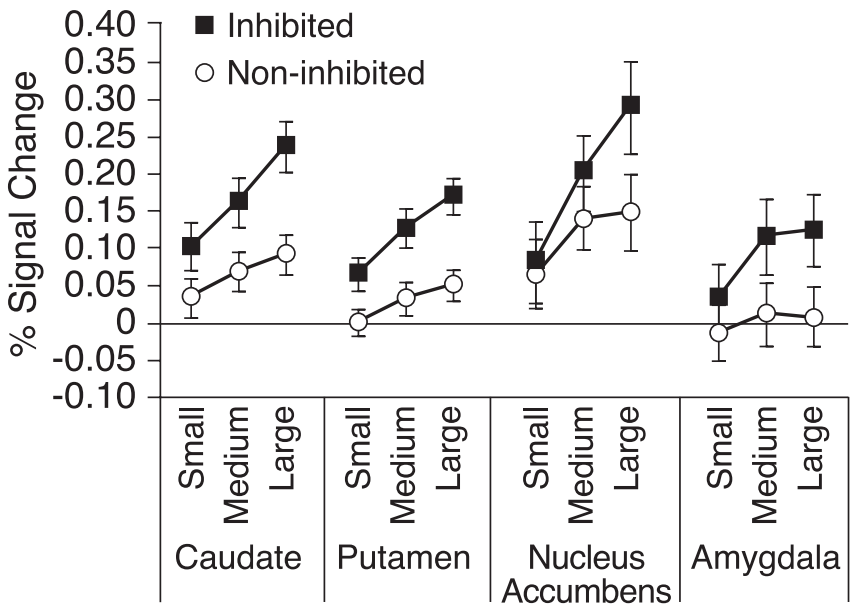

Figure 2. Between-group comparisons of event-elicited percentage BOLD signal change in the caudate, putamen, nucleus accumbens, and amygdala. Data were averaged within each ROI and extracted at fMRI acquisition during anticipation of large, medium, and small incentives collapsed across loss and gain trials. Error bars represent SEM.

and nucleus accumbens (mean \pm SE, $0.15 \pm 0.03 ; p<0.001$ ) than in the putamen (mean \pm SE, $0.07 \pm 0.01)$. The caudate and nucleus accumbens demonstrated similar levels of activation. Because there was not a significant group $\times$ region effect, no additional analyses were conducted to identify specific group-related patterns of response. As an example, Figure 3 shows the group differences in caudate activation at each incentive magnitude on reward trials.

Because the sample included prepubertal and postpubertal subjects and because sex and age differences have been noted in striatal dopamine receptors (Andersen et al., 1997; Becker, 1999; Mozley et al., 2001; Andersen et al., 2002), Figure 4 presents a scatter plot to illustrate the distribution of sex, age group, and inhibition status in relation to striatal activation at each incentive magnitude. Percentage signal change was averaged for gain and loss cues within the caudate, putamen, and nucleus accumbens separately at small, medium, and large incentives. Averages signal change values were $z$ scored and then summed across regions. Sex and age group were not significantly associated with striatal activation at any of the incentive levels (all $r$ values $<0.10$ ), whereas behavioral inhibition status correlated significantly with greater striatal activity during anticipation of medium $(r=0.34 ; p=$ $0.04)$ and large $(r=0.51 ; p=0.003)$ incentives.

With regard to the amygdala, activation was not influenced by the interaction of group with valence or incentive magnitude. However, the amygdala was sensitive to the different incentive magnitudes $\left(F_{(2,60)}=6.66 ; p<0.005\right)$. Specifically, amygdala activation was greater during anticipation of the large (mean \pm $\mathrm{SE}, 0.07 \pm 0.03 ; p=0.02$ ) and medium (mean $\pm \mathrm{SE}, 0.06 \pm 0.03$; $p=0.01$ ) incentives relative to the small incentive (mean $\pm \mathrm{SE}$, $0.01 \pm 0.03$ ). Amygdala activation did not differ as a function of valence.

The analysis involving BA17 showed no significant main or interaction effects of group, valence, or incentive magnitude. The lack of activation in BA17 in response to the MID is consistent with our expectation of specific task-related regional activation. A group effect $\left(F_{(1,30)}=8.44 ; p<0.01\right)$ and an incentive effect $\left(F_{(2,60)}=11.06 ; p<0.001\right)$ were found on BA4 activation. Specifically, BA4 activity was greater for the BI group (mean $\pm \mathrm{SE}$, $0.11 \pm 0.02$ ) versus $\mathrm{BN}$ group (mean $\pm \mathrm{SE}, 0.02 \pm 0.02$ ) and during anticipation of the large (mean \pm SE, $0.10 \pm 0.02 ; p<$
$0.001)$ and medium (mean $\pm \mathrm{SE}, 0.07 \pm 0.02 ; p=0.03)$ incentives relative to the small incentive (mean $\pm \mathrm{SE}, 0.03 \pm 0.02$ ). No interaction effects were found. Thus, between-group differences in BA4 activity were not moderated by incentive salience or cue valence.

\section{Discussion}

The present study showed that adolescents previously classified as behaviorally inhibited in early childhood demonstrated enhanced sensitivity to incentives relative to adolescents not classified as such. As hypothesized, enhanced sensitivity was manifest as greater striatal activation while anticipating monetary gain or loss. Importantly, striatal activation differed between groups as a function of incentive magnitude. These group differences emerged despite comparable behavioral performances.

The current findings relate evaluation of stimulus salience to behavioral inhibition. We view salience as a quantifiable value that influences motivation to act on a stimulus. Previous studies show that stimulus salience influences striatal response to rewards (Zink et al., 2003, 2004, 2006). For example, Zink et al. (2004) found that striatal activation depends on the salience of rewards rather than their value. Other work associates striatal response with performance of actions aimed at reward acquisition (Tricomi et al., 2004). The present study related betweensubject variation in behavioral inhibition to between-subject variation in striatal activation.

Our second hypothesis regarding amygdala response was not confirmed; we found differences in amygdala activation based on incentive magnitude but not based on inhibition status. To date, the only other fMRI study to examine temperamentally based group differences found enhanced amygdala activity in response to novel, neutral face stimuli in adults who had been classified as inhibited as toddlers compared with adults who were not previously classified as such (Schwartz et al., 2003). The lack of between-group differences in the present study may indicate that the MID loss stimuli were not novel enough to elicit greater amygdala activation in the behaviorally inhibited group as demonstrated previously (Schwartz et al., 2003). Nonetheless, findings from both studies suggest that the early temperamental disposition of behavioral inhibition is associated with enhanced sensitivity in multiple neural regions that process diverse types of salient stimuli.

Behaviorally, we did not find group differences in MID performance. This was expected because, by design, difficulty was adjusted to match subject ability. In previous work, however, we documented greater sensitivity to rewards in shy versus non-shy college students, as indexed by reaction time (Hardin et al., 2006). The discrepancy between studies may be related to a number of factors, including the following: (1) experimental conditions, such as fMRI environment; (2) manipulation of target duration in the present study but not in our behavioral study; (3) parameterization of the present MID task; (4) smaller sample size; (5) age of samples; and (6) a different measure of temperament used to define the groups. Nonetheless, our neural findings are consistent with the behavioral finding of Hardin et al. (2006), i.e., enhanced response to reward cues in shy/behaviorally inhibited subjects compared with non-shy/non-inhibited subjects.

Two exploratory findings also emerged from the present study. First, although valence modulated striatal activity (i.e., greater activation in response to gain vs loss cues) across groups, it did not differentially affect the groups. The prominence of gain versus loss in driving striatal activity is consistent with previous work using the MID task (Knutson et al., 2001a; Bjork et al., 


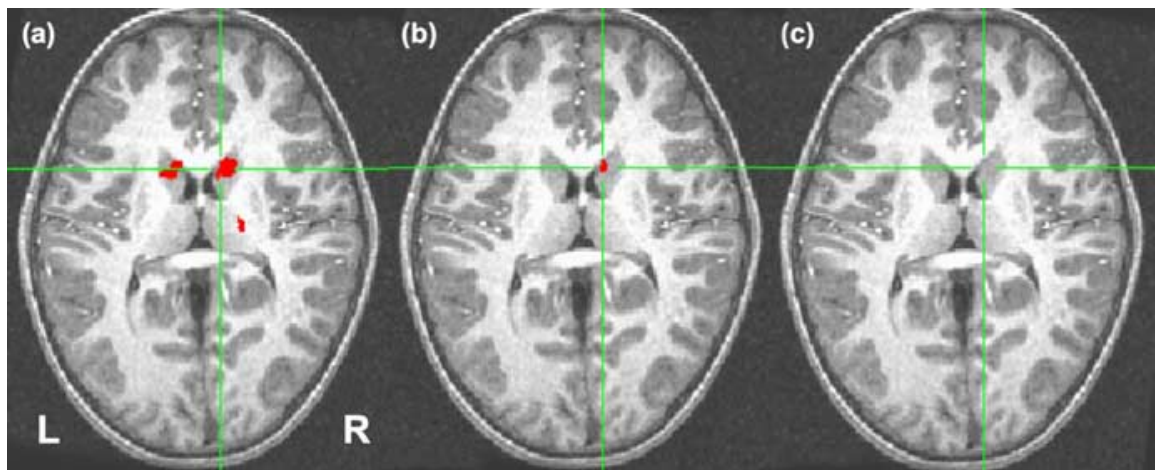

Figure 3. Statistical maps centered in the right caudate (threshold, 3.64; $p<0.001$, whole-brain uncorrected; $x=11, y=11$, $z=13$ ) of voxelwise between-group comparisons during anticipation at each incentive that could be won. $\boldsymbol{a}, \boldsymbol{b}, \mathrm{BI}$ adolescents compared with BN adolescents exhibited greater activation to large gain versus no gain in the left $(\mathrm{L})$ and right $(\mathrm{R})$ caudate $(\boldsymbol{a})$ and greater activation to medium gain versus no gain in the right caudate $(\boldsymbol{b})$. $\boldsymbol{c}$, No group differences emerged in caudate activation during anticipation of small gain versus no gain.
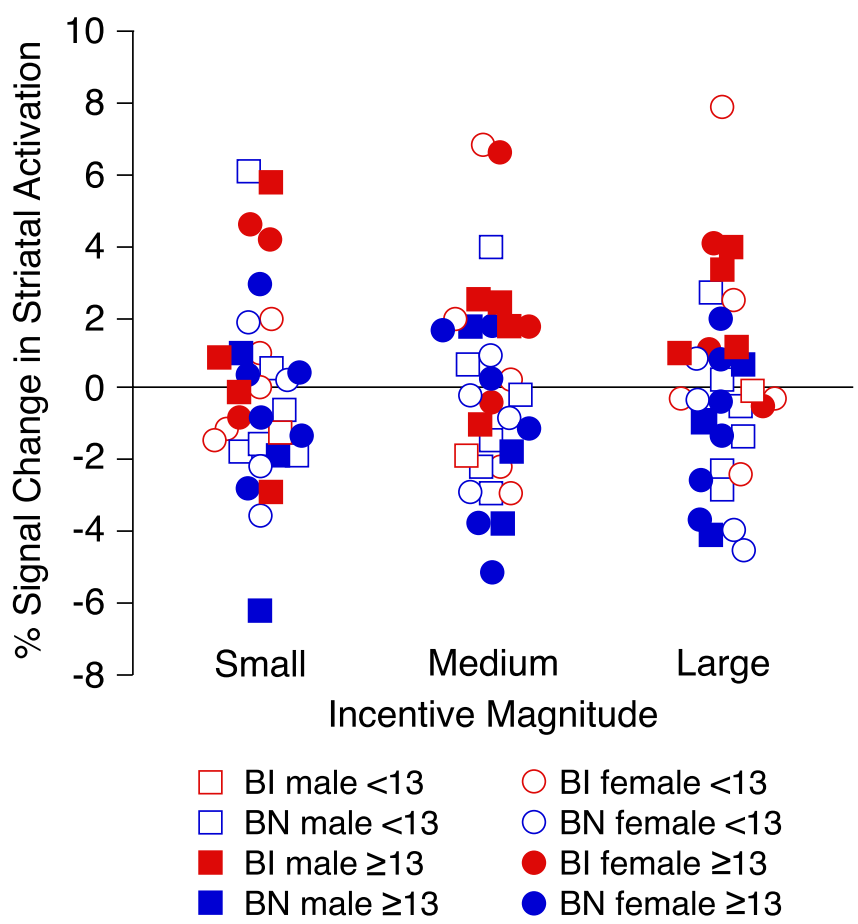

Figure 4. Scatter plot depicting percentage signal change in striatal activation for subjects by inhibition status, sex, and age group (before or after 13 years of age). Percentage signal change was averaged for gain and loss cues within the caudate, putamen, and nucleus accumbens separately at small, medium, and large incentive magnitudes. Average signal change values were $z$ scored and then summed across regions.

2004). Second, group differences in striatal activation were not regionally specific because both groups showed greater activity in the caudate and nucleus accumbens compared with the putamen. The general prominence of caudate and nucleus accumbens activation to incentives is consistent with studies using the present MID task (Knutson et al., 2001a) as well as studies using other reward paradigms (Delgado et al., 2003; Tricomi et al., 2004; Zink et al., 2004). A recent study attempted to dissociate the rewardrelated function of caudate/nucleus accumbens from that of the putamen (Haruno and Kawato, 2006). The authors found that caudate and nucleus accumbens activity correlated with rewardprediction errors, whereas putamen activity correlated with the degree to which reward associations were learned and realized, i.e., stimulus-action reward (Haruno and Kawato, 2006). By inference, greater activity in the caudate and nucleus accumbens during the MID may reflect task characteristics that more specifically probe the uncertainty of reinforcement and rewardprediction errors than the execution of a motivated action. Currently, data in humans on the specific role of various striatal regions in reward processes remain preliminary.

One caveat of the present findings is that enhanced striatal activation in the behaviorally inhibited group relative to the non-inhibited group could also reflect a nonspecific, generalized state of higher arousal in anxiety-prone subjects. Although this possibility cannot be ruled out, the absence of group differences in primary visual cortex activity, which is sensitive to attention states (Astafiev et al., 2004; Kastner and Pinsk, 2004), argues against it.

Observed striatal differences may indicate increased activation in a distributed motor circuit. Consistent with this possibility, the behaviorally inhibited group showed greater motor cortex activity than the non-inhibited group. These findings are compatible with past work documenting higher muscle tension in inhibited versus non-inhibited children (Kagan, 1994). Moreover, in both behaviorally inhibited and non-inhibited subjects, greater motor cortex activity was also found during anticipation of the larger incentives. This pattern is consistent with previous neuroimaging work using the MID task in adults (Knutson et al., 2000). By inference, these data suggest that both behavioral inhibition and stimulus salience influence functioning in corticostriatal motor circuitry (Hikosaka et al., 1989). The absence of group $\times$ incentive or group $\times$ valence interactions, however, indicates that between-group differences in motor cortex activation are not modulated by incentive magnitude or cue valence. Thus, group $\times$ incentive interactions are limited to the striatum.

One limitation of the present study is the relatively small sample size. Although the groups did not differ significantly in sex and age, our sample size did not permit a full test of whether temperamentally based group differences in striatal response to incentives are further influenced by sex and age/pubertal status. We also note that, although sex- and age-related differences in dopamine levels may influence striatal function (Andersen et al., 1997; Becker, 1999; Mozley et al., 2001; Andersen et al., 2002), the direction of these influences is not well known in human adolescent samples. Thus, future studies using larger adolescent samples are necessary to tease apart these potential influences on the relationship between striatal function and behavioral inhibition.

Both behavioral [e.g., reticence with peers (Rubin et al., 2002)] and physiological [e.g., right frontal EEG asymmetry (Fox et al., 1995)] response profiles documented in inhibited children early in life suggest that this form of temperament is characterized by a stable, general hypersensitivity to environmental stimuli that has typically been understood in terms of enhanced reactivity of the fear circuitry of the brain to novel stimuli (Kagan, 1994; Schwartz et al., 2003; Fox et al., 2005). The main finding from the current study extends this characterization by demonstrating that an inhibited temperament is associated with enhanced neural sensitivity in brain regions that facilitate motivated behavior in response to nonsocial reward stimuli. One interpretation of 
the present findings is that individuals with behavioral inhibition may have heightened striatal activation because they are concerned about making errors, particularly as the stakes increase with larger incentives. The greater incentive-related increase in striatal activation in the behaviorally inhibited group may indicate that individuals who experience substantial worry or anxiety exhibit increased motivation and enhanced vigilance in response to highly salient stimuli, perhaps energized by a strong desire to avoid failure (Eysenck and Calvo, 1992). Previous work demonstrating enhanced error monitoring in behaviorally inhibited children supports this possibility (Henderson, 2003). Based on inhibited children's reactions to unfamiliar adults and peers (Kagan et al., 1988b; Rubin et al., 2002), future work might consider whether this sensitivity to nonsocial rewards extends to social rewards as well.

The current findings may signify a developing vulnerability for later psychopathology associated with behavioral inhibition that is characterized by functional anomalies in incentive processing. Indeed, research on behavioral inhibition and psychopathology has emphasized a relationship with both anxiety disorders (Hirshfeld et al., 1992; Biederman et al., 1993, 2001; Schwartz et al., 1999) and depression (Caspi et al., 1996; Rosenbaum et al., 2000). Documenting between-group differences in behavioral tendencies, such as temperament and personality traits, as predictors of neural response is important for identifying individuals who may be at risk for developing psychopathology (Canli et al., 2002). A recent study using the MID found reduced striatal activity during reward anticipation in adolescents with attention deficit hyperactivity disorder (ADHD) compared with healthy adolescents (Scheres et al., 2006). These data implicate diminished salience of anticipated rewards in ADHD, findings opposite to those from our study revealing enhanced sensitivity in behaviorally inhibited adolescents. Such data are consistent with other work examining the relationship between behavioral disinhibition and ADHD (Hirshfeld-Becker et al., 2002). Together, these studies suggest that research on betweengroup differences in neural response to incentives may elucidate underlying substrates of specific adolescent psychopathologies (Ernst et al., 2006). Thus, the present findings offer a possible vulnerability marker that could guide future neuroimaging studies on individual differences associated with risk for specific psychopathologies.

\section{References}

Achenbach TM, Howell CT, Quay HC, Conners CK (1991) National survey of problems and competencies among four- to sixteen-year-olds: parents' reports for normative and clinical samples. Monogr Soc Res Child Dev $56: 1-131$

Andersen SL, Rutstein M, Benzo JM, Hostetter JC, Teicher MH (1997) Sex differences in dopamine receptor overproduction and elimination. NeuroReport 8:1495-1498.

Andersen SL, Thompson AP, Krenzel E, Teicher MH (2002) Pubertal changes in gonadal hormones do not underlie adolescent dopamine receptor overproduction. Psychoneuroendocrinology 27:683-691.

Astafiev SV, Stanley CM, Shulman GL, Corbetta M (2004) Extrastriate body area in human occipital cortex responds to the performance of motor actions. Nat Neurosci 7:542-548.

Baxter MG, Parker A, Lindner CC, Izquierdo AD, Murray EA (2000) Control of response selection by reinforcer value requires interaction of amygdala and orbital prefrontal cortex. J Neurosci 20:4311-4319.

Becker JB (1999) Gender differences in dopaminergic function in striatum and nucleus accumbens. Pharmacol Biochem Behav 64:803-812.

Berns GS, McClure SM, Pagnoni G, Montague PR (2001) Predictability modulates human brain response to reward. J Neurosci 21:2793-2798.

Biederman J, Rosenbaum JF, Bolduc-Murphy EA, Faraone SV, Chaloff J, Hirshfeld DR, Kagan J (1993) A 3-year follow-up of children with and without behavioral inhibition. J Am Acad Child Adolesc Psychiatry 32:814-821.

Biederman J, Hirshfeld-Becker DR, Rosenbaum JF, Herot C, Friedman D, Snidman N, Kagan J, Faraone SV (2001) Further evidence of association between behavioral inhibition and social anxiety in children. Am J Psychiatry 158:1673-1679.

Bjork JM, Knutson B, Fong GW, Caggiano DM, Bennett SM, Hommer DW (2004) Incentive-elicited brain activation in adolescents: similarities and differences from young adults. J Neurosci 24:1793-1802.

Breiter HC, Aharon I, Kahneman D, Dale A, Shizgal P (2001) Functional imaging of neural responses to expectancy and experience of monetary gains and losses. Neuron 30:619-639.

Buss AH, Plomin R (1984) Temperament: early personality traits. Hillsdale, NJ: Erlbaum.

Cairns RB, Bergman LR, Kagan J (1998) Methods and models for studying the individual. Thousand Oaks, CA: Sage.

Calkins SD, Fox NA, Marshall TR (1996) Behavioral and physiological antecedents of inhibited and uninhibited behavior. Child Dev 67:523-540.

Canli T, Sivers H, Whitfield SL, Gotlib IH, Gabrieli JD (2002) Amygdala response to happy faces as a function of extraversion. Science 296:2191.

Caspi A, Moffitt TE, Newman DL, Silva PA (1996) Behavioral observations at age 3 years predict adult psychiatric disorders: longitudinal evidence from a birth cohort. Arch Gen Psychiatry 53:1033-1039.

Cohen MS (1997) Parametric analysis of fMRI data using linear systems methods. NeuroImage 6:93-103.

Cox RW (1996) AFNI: software for analysis and visualization of functional magnetic resonance neuroimages. Comput Biomed Res 29:162-173.

Daw ND, Niv Y, Dayan P (2005) Uncertainty-based competition between prefrontal and dorsolateral striatal systems for behavioral control. Nat Neurosci 8:1704-1711.

Delgado MR, Locke HM, Stenger VA, Fiez JA (2003) Dorsal striatum responses to reward and punishment: effects of valence and magnitude manipulations. Cogn Affect Behav Neurosci 3:27-38.

Ernst M, Nelson EE, Jazbec S, McClure EB, Monk CS, Leibenluft E, Blair J, Pine DS (2005) Amygdala and nucleus accumbens in responses to receipt and omission of gains in adults and adolescents. NeuroImage 25:1279-1291.

Ernst M, Pine DS, Hardin M (2006) Triadic model of the neurobiology of motivated behavior in adolescence. Psychol Med 36:299-312.

Eysenck MW, Calvo MG (1992) Anxiety and performance: the processing efficiency theory. Cognit Emotion 6:409-434.

Fox NA, Rubin KH, Calkins SD, Marshall TR, Coplan RJ, Porges SW, Long JM, Stewart S (1995) Frontal activation asymmetry and social competence at four years of age. Child Dev 66:1770-1784.

Fox NA, Schmidt LA, Calkins SD, Rubin KH, Coplan RJ (1996) The role of frontal activation in the regulation and dysregulation of social behavior during the preschool years. Dev Psychopathol 8:89-102.

Fox NA, Henderson HA, Rubin KH, Calkins SD, Schmidt LA (2001) Continuity and discontinuity of behavioral inhibition and exuberance: Psychophysiological and behavioral influences across the first four years of life. Child Dev 72:1-21.

Fox NA, Henderson HA, Marshall PJ, Nichols KE, Ghera MM (2005) Behavioral inhibition: linking biology and behavior within a developmental framework. Annu Rev Psychol 56:235-262.

Hardin MG, Perez-Edgar K, Guyer AE, Pine DS, Fox NA, Ernst M (2006) Reward and punishment sensitivity in shy and exuberant adults: a relationship between social and motivated behavior. Pers Individ Dif 40:699-711.

Haruno M, Kawato M (2006) Different neural correlates of reward expectation and reward expectation error in the putamen and caudate nucleus during stimulus-action-reward association learning. J Neurophysiol 95:948-959.

Henderson HA (2003) Temperamental contributions to problem solving: cognitive and affective processes. Dissert Abstr Int B Sci Engin 64:441.

Henderson HA, Marshall PJ, Fox NA, Rubin KH (2004) Psychophysiologi$\mathrm{cal}$ and behavioral evidence for varying forms and functions of nonsocial behavior in preschoolers. Child Dev 75:251-263.

Hikosaka O, Sakamoto M, Usui S (1989) Functional properties of monkey caudate neurons. III. Activities related to expectation of target and reward. J Neurophysiol 61:814-832.

Hirshfeld-Becker DR, Biederman J, Faraone SV, Violette H, Wrightsman J, Rosenbaum JF (2002) Temperamental correlates of disruptive behavior 
disorders in young children: preliminary findings. Biol Psychiatry 51:563-574.

Hirshfeld DR, Rosenbaum JF, Biederman J, Bolduc EA, Faraone SV, Snidman N, Reznick JS, Kagan J (1992) Stable behavioral inhibition and its association with anxiety disorder. J Am Acad Child Adolesc Psychiatry 31:103-111.

Kagan J (1994) Galen's prophecy: temperament in human nature. New York: BasicBooks.

Kagan J, Snidman N (1991) Temperamental factors in human development. Am Psychol 46:856-862.

Kagan J, Reznick JS, Snidman N (1987) The physiology and psychology of behavioral inhibition in children. Child Dev 58:1459-1473.

Kagan J, Reznick JS, Snidman N (1988a) Biological bases of childhood shyness. Science 240:167-171.

Kagan J, Reznick JS, Snidman N, Gibbons J, Johnson MO (1988b) Childhood derivatives of inhibition and lack of inhibition to the unfamiliar. Child Dev 59:1580-1589.

Kastner S, Pinsk MA (2004) Visual attention as a multilevel selection process. Cogn Affect Behav Neurosci 4:483-500.

Kaufman J, Birmaher B, Brent D, Rao U, Flynn C, Moreci P, Williamson D, Ryan N (1997) Schedule for affective disorders and schizophrenia for school-age children-present and lifetime version (K-SADS-PL): initial reliability and validity data. J Am Acad Child Adolesc Psychiatry 36:980-988.

Knutson B, Westdorp A, Kaiser E, Hommer D (2000) FMRI visualization of brain activity during a monetary incentive delay task. NeuroImage 12:20-27.

Knutson B, Adams CM, Fong GW, Hommer D (2001a) Anticipation of increasing monetary reward selectively recruits nucleus accumbens. J Neurosci 21:RC159(1-5).

Knutson B, Fong GW, Adams CM, Varner JL, Hommer D (2001b) Dissociation of reward anticipation and outcome with event-related fMRI. NeuroReport 12:3683-3687.

LeDoux JE, Iwata J, Cicchetti P, Reis DJ (1988) Different projections of the central amygdaloid nucleus mediate autonomic and behavioral correlates of conditioned fear. J Neurosci 8:2517-2529.

Mozley LH, Gur RC, Mozley PD, Gur RE (2001) Striatal dopamine transporters and cognitive functioning in healthy men and women. Am J Psychiatry 158:1492-1499.

Neter J, Kutner MH, Machtsheim CJ, Wasserman W (1996) Applied linear statistical models, Ed 4. Chicago: Irwin.
O’Doherty J, Dayan P, Schultz J, Deichmann R, Friston K, Dolan RJ (2004) Dissociable roles of ventral and dorsal striatum in instrumental conditioning. Science 304:452-454.

Reynolds SM, Berridge KC (2002) Positive and negative motivation in nucleus accumbens shell: bivalent rostrocaudal gradients for GABA-elicited eating, taste "liking"/“disliking" reactions, place preference/avoidance, and fear. J Neurosci 22:7308-7320.

Roitman MF, Wheeler RA, Carelli RM (2005) Nucleus accumbens neurons are innately tuned for rewarding and aversive taste stimuli, encode their predictors, and are linked to motor output. Neuron 45:587-597.

Rosenbaum JF, Biederman J, Hirshfeld-Becker DR (2000) A controlled study of behavioral inhibition in children of parents with panic disorder and depression. Am J Psychiatry 157:2002-2010.

Rothbart MK, Ahadi SA, Hershey KL, Fisher P (2001) Investigations of temperament at three to seven years: the Children's Behavior Questionnaire. Child Dev 72:1394-1408.

Rubin KH, Burgess KB, Hastings PD (2002) Stability and social-behavioral consequences of toddlers' inhibited temperament and parenting behaviors. Child Dev 73:483-495.

Scheres A, Milham MP, Knutson B, Castellanos FX (2006) Ventral striatal hyporesponsiveness during reward anticipation in Attention-Deficit/Hyperactivity Disorder. Biol Psychiatry, in press.

Schwartz CE, Snidman N, Kagan J (1999) Adolescent social anxiety as an outcome of inhibited temperament in childhood. J Am Acad Child Adolesc Psychiatry 38:1008-1015.

Schwartz CE, Wright CI, Shin LM, Kagan J, Rauch SL (2003) Inhibited and uninhibited infants "grown up": adult amygdalar response to novelty. Science 300:1952-1953.

Talairach J, Tournoux P (1988) Co-planar stereotaxic atlas of the human brain. Stuttgart, Germany: Thieme Verlag.

Tricomi EM, Delgado MR, Fiez JA (2004) Modulation of caudate activity by action contingency. Neuron 41:281-292.

Zink CF, Pagnoni G, Martin ME, Dhamala M, Berns GS (2003) Human striatal response to salient nonrewarding stimuli. J Neurosci 23:8092-8097.

Zink CF, Pagnoni G, Martin-Skurski ME, Chappelow JC, Berns GS (2004) Human striatal responses to monetary reward depend on saliency. Neuron 42:509-517.

Zink CF, Pagnoni G, Chappelow J, Martin-Skurski M, Berns GS (2006) Human striatal activation reflects degree of stimulus saliency. NeuroImage 29:977-983. 